\title{
O legado de Feynman visto por pesquisadores brasileiros
}

Feynman's legacy seen by Brazilian researchers

\author{
Nelson Studart*1,2 \\ ${ }^{1}$ Universidade Federal do ABC, Centro de Ciências Naturais e Humanas, Santo André, SP, Brasil \\ ${ }^{2}$ Universidade Federal de São Carlos, São Carlos, SP, Brasil
}

Recebido em 28 de Maio, 2018. Aceito em 29 de Maio, 2018.

\begin{abstract}
Esse artigo apresenta os ensaios da edição especial da Revista Brasileira de Ensino de Física, comemorativa do centenário de nascimento de Richard P. Feynman. A partir de um depoimento pessoal de sua descoberta de Feynman, o autor busca complementar, com um pouco de história, os artigos de pesquisadores brasileiros convidados, que refletem suas visões sobre a física e o ensino de física, para as quais Feynman contribuiu decisivamente.
\end{abstract}

Palavras-chave: Feynman, memorial, física quântica, ensino de física.

This article presents the essays of the special edition of the Revista Brasileira de Ensino de Física, celebrating the centenary of Richard P. Feynman's birth. From a personal testimony of his discovery of Feynman, the author seeks to complement, with few historical remarks, the articles of the invited Brazilian researchers, which reflect their views on physics and physics teaching, to which Feynman contributed decisively.

Keywords: Feynman, memorial, quantum physics; physics teaching.

He believed in the primacy of doubt; not as a blemish upon on our ability to

know but as the essence of knowing. James Gleick

\section{Introdução}

Richard Phillips Feynman (1918-1988) foi uma brilhante mente científica. Suas contribuições à Física e ao seu ensino são reconhecidas como fundamentais; Feynman é reconhecido por seus pares como um dos mais importantes físicos do século XX. Seus epítetos mais conhecidos são gênio, excêntrico, perspicaz, irônico; na opinião de Bill Gates, é "phenomenal explainer, amazing scientist, and all-around colorful guy" e o mais destacado, "curious character". Freeman Dyson o descreveu como "half genius and half buffoon, who keeps all physicists and their children with his effervescent vitality" [1]. Além de todos os atributos que lhe são conferidos, Feynman é hoje uma figura midiática com enorme repercussão no mundo virtual. Uma busca de Feynman revela mais de 6 milhões de resultados no Google e mais de 250 mil no Youtube, onde se destacam os vídeos de suas monumentais palestras. Seus livros de Física e de divulgação científica - poucos escritos por ele mesmo - são enorme sucesso em todo o mundo.

A Revista Brasileira de Ensino de Física reverencia o cientista Feynman por ocasião do centenário de seu nas-

*Endereço de correspondência: n.studart@gmail.com cimento com essa edição especial retratando seu legado na visão de pesquisadores brasileiros.

Essa apresentação começa com um depoimento pessoal. Ainda estudante no Instituto de Física da UFC, fui apresentado a Feynman por meio das notas de aula de um minicurso "Formulação de Integrais de Trajetória da Mecânica Quântica", escritas por Luís Carlos Gomes, baseado no livro de Feynman e seu aluno Hibbs. [2,3] Não entendi quase nada, mas era tudo muito diferente do que tinha visto até então sobre o tema. Ao final da graduação, o livro-texto da disciplina Mecânica Quântica, oferecida por Homero Lenz Cesar, foi o volume III do Lectures on Physics. [4] Fui seduzido pela abordagem de Feynman, pela maneira original de explicar a dualidade onda-partícula por meio da experiência de dupla-fenda, de introduzir sistemas de dois estados via o maser de amônia, e de usar o aparato de Stern-Gerlach para "descrever um fenômeno quântico de uma maneira completamente quântica", sem ligações com a mecânica clássica, como usual nos textos tradicionais. Enfim, para mim, tudo tremendamente original.

Quanto estive na $\mathrm{UnB}$, tomei conhecimento de que o volume III era adotado desde a criação do curso de física, ministrado com entusiasmo por Fernando de Sousa 
Barros que sugeria que os alunos usassem o livro como travesseiro apenas para lembrar quando acordassem que deveriam continuar leitura 1

Anos depois, tive o privilégio de assistir a seu colóquio Negative Probabilities no MIT, em 1981, que se transformou em artigo em Simpósio em homenagem a David Bohm. [5] onde se lê: "Unfortunately, I never did find out how to use the freedom of allowing probabilities to be negative to solve the original problem of infinities in quantum field theory". Entretanto, ele discute alguns exemplos físicos em que as probabilidades são negativas em eventos intermediários. Fiquei fascinado pelo seu modo de falar e pelas expressões faciais e gestuais, que tornavam as explicações se não tão inteligíveis pela complexidade do tema, pelo menos, extremamente atraentes.

Durante o doutorado, tive contato com o problema de pólarons em aulas do Roberto Lobo no IFSC-USP, acerca das integrais de trajetória e tive a ideia da extensão da solução de Feynman do problema do pólaron ao que veio a se chamar pólaron riplônico, em que ríplons nos filmes de hélio líquido fazem o papel dos fônons em cristais iônicos. Trato desse tema em artigo dessa edição.

Considero um primor suas Messenger Lectures, tendo contribuído para a tradução da versão impressa [6] dessas maravilhosas palestras agora disponíveis em vídeo pelo projeto Tuva da Microsoft.

Feynman foi e continua sendo um dos meus ídolos e permanente fonte de inspiração. Aprecio seus livros técnicos de Mecânica Quântica e de Mecânica Estatística, e a maioria dos livros de divulgação científica, inclusive aquele em que narra suas memórias a Ralph Leighton, apesar de pouca generosidade com o Brasil de que, apesar do escrito, gostava muito. [7]. As histórias contadas revelam aspectos de seu caráter irônico, irreverente, burlesco e agressivo com palestrantes convidados. Possuía autoestima elevada beirando a autopromoção, como realçado pelo comentário do seu colega e competidor, Murray Gell-Mann: "Feynman was a great scientist, but he spent a great deal of his effort generating anecdotes about himself".

Os ensaios, escritos a convite nessa edição especial da RBEF, estão distribuídos em seções temáticas. A Seção 2, Feynman e o ensino de física, aborda sua influência no ensino de Física tanto na produção de material instrucional quanto na crítica contundente à maneira como a física era ensinada. Nestor Caticha, do IFUSP, rememora as aulas de Feynman e o ambiente da pós-graduação do Caltech nos anos 80. Ildeu de Castro Moreira da UFRJ narra as visitas de Feynman ao Brasil e sua marcante influência no ensino de Física na América Latina e Ricardo Karam da Universidade de Copenhague analisa a abordagem de Feynman em suas Lectures e o que ela apresenta de diferente quando comparada com livros tradicionais usados

\footnotetext{
1 J.M.F. Bassalo conta esse episódio no artigo comemorativo de seus 25 anos de doutorado, publicado em Ciência e Sociedade, do CBPF, número 3 de 2000.
}

em disciplinas introdutórias de física. A Seção 3, Feynman e a quântica, discute algumas de suas contribuições inovadoras e fundamentais na área: a proposta de uma nova formulação da mecânica quântica em termos de integrais de caminhos, apresentada por David Vianna do IFUFBA; a revolucionária criação dos agora famosos diagramas de Feynman, exposto com clareza pela Cristina Aguilar do IFGW-Unicamp; e algumas conexões entre a Mecânica Quântica e a Clássica, elaboradas com rigor conceitual pelo Alfredo Osório de Almeida do CBPF. Vicente Pleitez, do IFT-Unesp, detalha as origens e os caminhos que levaram Feynman, Schwinger e Tomonaga a estabelecer a teoria quântica da eletrodinâmica, um dos maiores avanços no conhecimento das interações fundamentais; essa seção ainda contém a palestra de Feynman ao receber o Nobel de Física, traduzida por Marcel Novaes, do IF-UFU. Na Seção 4, encontra-se o Feynman visionário, em que Peter Schulz, da FCA-Unicamp, analisa a célebre conferência de Feynman anunciando que dimensões na escala quântica poderiam ser alcançadas, criando cenário propício para a nanociência e a nanotecnologia. Além disso, Amir Caldeira, do IFGW-Unicamp aponta as contribuições de Feynman para dois ramos da física que foram desenvolvidos independentemente, mas que se tornaram essenciais para a desenvolvimento do processador quântico: os efeitos da mecânica quântica na teoria da computação e a coerência quântica. Na Seção 5, Feynman e a matéria condensada, Paulo Farinas, do DF-UFSCar, e o autor desta apresentação discutem as contribuições seminais de Feynman a dois problemas da física da matéria condensada: a teoria microscópica do hélio superfluido, em que complementou a teoria fenomenológica da Landau, e o tratamento dos pólarons em cristais, usando sua formulação de integrais de caminho, que abriu as portas para a sua aplicação em outros problemas da matéria condensada. Finalmente, na seção 6, Feynman e as partículas elementares, Carlos Escobar, do Fermilab, escreve sobre a invenção dos pártons como constituintes do próton. Na Seção 7, são apresentadas considerações gerais.

\section{Feynman e o ensino de Física}

No artigo inicial dessa seção Nestor Caticha relembra o ambiente no Caltech nos anos 80, e comenta seu prazer em assistir as aulas de Feynman e a procura dos alunos em cursar as disciplinas de pós-graduação dadas por ele. Além disso, apresenta deliciosas anedotas sobre Feynman. É bom que nossos leitores saibam que Feynman preparava cuidadosamente suas aulas e que, além do conteúdo ministrado, descrevia suas experiências pessoais, refletia sobre as discussões com os gênios da física e ficava preocupado se não fosse inquirido pelos alunos. Há vários depoimentos de ex-alunos de Feynman que podem complementar a experiência de Caticha. Em particular, recomendo a leitura das impressões de Leonard Mlodinow em sua convivência com Feynman durante seu 
pós-doutorado no Caltech [8] e das reminiscências de Tony Hey de sua vida como pós-doc no grupo de Murray e Dick nos anos 70. [9] Outro depoimento interessante foi dado por Laurie Brown que foi orientado por Feynman em sua fase de Cornell. [10]

Feynman tinha enorme prazer em ministrar aulas, palestras e explicar coisas. Um dos seus apelidos é "Grande Explicador", atribuído pela sua capacidade de apresentar temas complexos numa linguagem simples. Ele também gostava muito de professores de ciências. Numa conferência em que pretendeu responder à pergunta What's Science?, ele mandou uma mensagem de entusiasmo e respeito pelos professores de ciência, possivelmente motivado pela admiração a seu pai, por lhe ensinar a questionar os fatos em ciência, explícita nessa mesma conferência:

"In the first place, from the way that I am preparing to give this lecture, it may seem that I am trying to tell you how to teach science - I am not at all in any way, because I don't know anything about small children. I have one, so I know that I don't know. The other is I think that most of you-because there is so much talk and so many papers and so many experts in the field-have some kind of a feeling of lack of self-confidence. In some way, you are always being lectured on how things are not going too well and how you should learn to teach better. I am not going to berate you for the bad work you are doing and indicate how it can definitely be improved; that is not my intention."[11]

Feynman tinha uma forma peculiar de estudar anotando no seu caderno de notas.

James Gleick2 anotou: "He opened a fresh notebook. On the title page he wrote: Notebook of things I don't know about. For the first but not last time he reorganized his knowledge. He worked for weeks at disassembling each branch of physics, oiling the parts, and putting them back together, looking all the while for the raw edges and inconsistencies. He tried to find the essential kernels of each subject."[12] Dado um certo conhecimento adquirido, Feynman ia separando-o em partes, diferenciando e esmerilhando os conceitos, procurando falhas no raciocínio e depois colocando tudo junto de volta. E o ciclo se repetia até se sentir seguro da aprendizagem. No final, tinha um caderno abrangente com assuntos que haviam sido desmembrados, esmiuçados e consolidados. É curioso que, de forma intuitiva, Feynman usava um dos pilares essenciais da aprendizagem significativa de Ausubel, a diferenciação progressiva e a reconciliação integradora, em que o

\footnotetext{
2 Esse costume perdurou para sempre em sua atividade acadêmica. Hey [9] refere-se ao "awkward Feynman notebooks" quando afirma que "after all, one could hardly publish a result that Feynman already knew about and had written down in his famous working notebooks but had not bothered to publish."
}

aluno vai diferenciando e integrando o conhecimento, e progressivamente construindo os chamados subsunçores, que não são definitivos pois podem sofrer novas diferenciações e integrações. [13] Em suma, a aprendizagem não é abrupta, mas progressiva. Infelizmente, nossa cultura de ensino é voltada para o treinamento para provas e exames, privilegiando a aprendizagem mecânica.

A influência de Feynman na educação e, em particular, no ensino de ciências, persiste até hoje. Ou melhor, tem se intensificado nos últimos tempos de discussões sobre mudanças de paradigmas na "ensinagem"(o processo de ensino e aprendizagem, tratado conjuntamente). Conhece-se hoje a chamada "técnica de Feynman", elaborada, segundo seus proponentes $[14,15]$, a partir de seu método de estudo, que consistiria em assimilar e transmitir conhecimento usando ideias concisas e linguagem simples. Trata-se de uma forma simplista que ignora a riqueza do pensamento de Feynman sobre como ensinar e como aprender.

No prefácio especial do "Lições de Física", [16] escrito por David Goodstein e Gerry Neugebauer, encontram-se duas pérolas de Feynman. A primeira tirada de rascunhos escritos no Brasil (1952):

"Em primeiro lugar, descubra por que quer que os alunos aprendam e o que quer que saibam e o método resultará mais ou menos por senso comum". [16, p. xii]

O "senso comum" para Feynman, segundo os autores, seriam "geralmente brilhantes reviravoltas que captavam com perfeição a essência de seu argumento". A segunda máxima pode ser extraída do insucesso de Feynman em não conseguir explicar para calouros por que partículas de spin $1 / 2$ obedecem à estatística de Fermi-Dirac e pode ser extrapolada para a necessidade de compreensão do conteúdo a ser ensinado: Se você não consegue fazer com que alunos entendam um determinado conceito que você está explicando é porque você realmente não o compreendeu!

No prefácio das "Lições de Física" [16], Feynman comenta sua concepção de "ensinagem." Teria sido Feynman um dos precursores das metodologias ativas? Ele critica o modelo tradicional (narrativa + resolução de problemas fechados) com a sugestão de um método interativo com foco na relação aluno-professor e a importância de um aluno ativo ao afirmar:

"Acredito porém que não há solução para esse problema de ordem educacional [fracasso dos alunos nos exames] a não ser abrir os olhos para o fato de que o ensino mais adequado só poderá ser levado a cabo nas situações em que houver um relacionamento pessoal direto entre o aluno e o bom professor - situações nas quais o estudante discuta as ideias, reflita e converse sobre elas. É impossível aprender muita coisa simplesmente comparecendo a 
uma palestra ou mesmo limitando-se a resolver os problemas determinados." [16, p. 5]

Outras valiosas informações sobre a pedagogia de Feynman expostas em suas duas conferências ministradas no Brasil, o leitor encontra na análise de Ildeu Moreira nessa edição. Uma delas, "O problema do ensino de Física na América Latina, foi publicada e a tradução está anexada. Feynman faz uma crítica contundente daquela aprendizagem mecânica que busca apenas a memorização de informações, sem margem para a reflexão e a construção de significados dos alunos.

Segundo David Goodstein e Gerry Neugebauer, "o próprio Feynman acreditava que sua maior contribuição para a física não seria a QED, a teoria do hélio superfluido, os pólarons ou os pártons. Sua mais notável contribuição seriam os três livros vermelhos das Feynman Lectures on Physics" [16, p. xiii]. Essa obra magistral e definitiva de Feynman é analisada por Ricardo Karam que aponta as peculiaridades da coleção que a tornam diferente dos livros-texto tradicionais de física introdutória. Karam responde positivamente à questão crucial: "Será que mesmo não sendo um Feynman, podemos extrair lições didáticas mais abrangentes das Lectures para sermos melhores professores de física?" Feynman teve uma visão pessimista sobre os efeitos positivos de suas palestras na aprendizagem dos alunos ingressantes no Caltech $3^{3}$ e mostrou-se preocupado com a evasão progressiva desse público almejado. No entanto, houve a adesão crescente de professores e estudantes de pós-graduação para suas palestras. Podese concluir com Karam que as Lectures são um precioso material instrucional para nossos professores de Física.

\section{Feynman e a quântica}

Feynman fez contribuições seminais em várias áreas da física, entre as quais se destacam a formulação de integrais de caminho da mecânica quântica, a teoria da eletrodinâmica quântica, a teoria da superfluidez do hélio líquido, a teoria das interações fracas, os limites fundamentais da computação, o modelo dos pártons como constituintes do próton. Exceto pelo seu papel na abordagem da interação V-A, feita com Murray Gell-Mann, essas contribuições são devidamente revistas nessa edição especial.

A formulação da mecânica quântica devido a Feynman é introduzida em palavras simples como ele gostava de se expressar: [17]

"Non-relativistic quantum mechanics is formulated here in a different way. It is, however, mathematically equivalent to the familiar formulation. In quantum mechanics the probability of an event, which can happen in several different ways, is the absolute square of a sum of complex contributions, one from

\footnotetext{
3 "Não acho que tenha me saído muito bem com os estudantes". Prefácio à edição de 1963.
}

each alternative way. The probability that a particle will be found to have a path $\mathrm{x}(\mathrm{t})$ lying somewhere within a region of spacetime is the square of a sum of contributions, one from each path in the region. The contribution from a single path is postulated to be an exponential whose (imaginary) phase is the classical action (in units of $\hbar$ ) for the path in question. The total contribution from all paths reaching $(\mathrm{x}, \mathrm{t})$ from the past is the wave function $\psi(\mathrm{x}, \mathrm{t})$. This is shown to satisfy Schroedinger's equation."

Mehra [18] nos conta que Feynman foi motivado inicialmente pelo "prato oscilante" (spinning-wobbling plate) e se interessou pelo problema das rotações de um elétron girante na relatividade e como tratá-lo usando integrais de caminho na mecânica quântica. Voltou-se para sua intuitiva formulação Lagrangeana da mecânica quântica proposta na sua tese de doutorado [20] e iniciou conversas com Dirac. Teve dificuldades em mostrar suas ideias em artigo, que continha parte de sua tese de doutorado (a qual não conseguiu publicar por causa do esforço de guerra em Los Alamos), mas foi encorajado por colegas. Em depoimento a Mehra, disse [18]:

"I wrote this paper and sent it to Physical Review, and it is suggested that I published in the Review of Modern Physics, which I did. At first, it was returned; they said it was too long, that was old hat, and that the first part in the paper was well known, which could be left out. Hans Bethe taught me a trick. He said, 'You have to emphasize that this part is known, and the others are new. It will take only a few paragraphs. In fact, I will shorten it for you.' Bethe took out a sentence, and said, 'If you make a small effort in this direction you don't have to take the whole thing.' That worked. They published it."

David Vianna mergulha no seu trabalho pioneiro, SpaceTime Approach to Non-Relativistic Quantum Mechanics, publicado no Reviews of Modern Physics, em 1948, mostrando em detalhes a construção da formulação da mecânica quântica usando integrais de trajetória. A influência de Dirac foi decisiva, uma vez que ele primeiro derivou uma relação entre o princípio de mínima ação e a mecânica quântica. Ele descreve sua caminhada rumo à construção dessa formulação na palestra Nobel, também nessa edição.

Os chamados diagramas de Feynman, representações pictóricas de expressões matemáticas complexas descrevendo processos de interação de objetos quânticos,

\footnotetext{
${ }_{4}$ Feynman, ao assumir o cargo de professor em Cornell, para se livrar de um estado depressivo, decidiu "brincar" com a física. Ao ver, na cantina, que um prato ao cair girava e oscilava, se propôs a resolver este problema, hoje conhecido como "Feynman's wobbling plate". [19]
} 
tornaram-se extremamente populares na comunidade internacional, embora nenhum físico tenha entendido o que Feynman estava fazendo com seus desenhos na sua primeira apresentação na Conferência de Pocono (1948). Pessoas com Bohr, Pauli e Dirac foram muito céticos achando que essa ferramenta de cálculo violava as bases da mecânica quântica ${ }^{5}$ David Kaiser [1] explica a razão por que os diagramas se espalharam rapidamente, apesar da compreensão inadequada inicial de muitos que o usaram. Ele aponta que Freeman Dyson foi o primeiro embaixador das ideias de Feynman escrevendo vários artigos esclarecendo pontos obscuros, mas o principal propagador (desculpe o trocadilho!) dos diagramas foi a educação pós-doutoral que começava a se estabelecer nos EUA. E Kaiser complementa: "since the middle of the 20th century, theoretical physicists have increasingly turned to this tool to help them undertake critical calculations", e "Feynman diagrams have revolutionized nearly every aspect of theoretical physics". Cristina Aguilar faz uma introdução breve, lúcida e palatável, dos diagramas de Feynman para o leitor interessado que nunca os usou.

A integral de caminho provê, em algum sentido, uma ponte entre a mecânica clássica e a mecânica quântica na formulação, devido a Feynman, já que "coloca de saída a ação clássica como o cerne da evolução", como nos explica Alfredo Osório de Almeida ao tratar da aproximação semiclássica na abordagem de Feynman e explorar a conexão clássica-quântica em sistemas periódicos: "Ao invés de apenas contrastar a multiplicidade de caminhos quânticos com a raridade das trajetórias clássicas, podemos nos valer do princípio variacional que as identificam para construir abrangentes aproximações semiclássicas para o operador de evolução."

A participação de Feynman na elaboração de Eletrodinâmica Quântica (QED), bem como no que consiste a teoria, é contada com riqueza de detalhes históricos pelo Vicente Pleitez. Quem desejar ir mais a fundo na história, sugiro o livro de Abraham Pais [21] e a notável biografia científica de Feynman por Jagdish Mehra. [18]

Não poderia estar ausente dessa coletânea de ensaios, a palestra de Feynman, na tradução de Marcel Novaes, ao receber o prêmio Nobel que, segundo Hey [10], deveria ser lida por todo aspirante a cientista porque "he described all the blind alleys and wrong ideas he had encountered on the ways to his great discoveries" ao afirmar um entusiasmo quase juvenil nessa metáfora: "Esse foi o começo, e a ideia me parecia tão óbvia e elegante que me apaixonei profundamente por ela. E isso, assim como se apaixonar por uma mulher, só é possível se você não souber muito sobre ela, de modo que não veja seus defeitos. Os defeitos vão acabar aparecendo, mas depois que o amor é forte o bastante para prendê-lo a ela." E

\footnotetext{
5 Dirac, apesar de seu bem conhecido mutismo, repetidamente interrogava sobre o que lhe parecia obscuro no esquema: a "unitariedade", relacionada com a conservação de probabilidade; as probabilidades de todos os resultados fisicamente possíveis devem totalizar a unidade. Feynman não conseguiu responder.
}

para os professores, ele revela uma dica para uma aula mais atraente: "Incluirei nas histórias detalhes que não têm valor científico, nem ajudam na compreensão das ideias. Serão incluídos apenas para tornar a palestra mais interessante."

\section{Feynman visionário}

O Feynman premonitório aparece nos dois artigos de Peter Schulz e Amir Caldeira tratando de suas contribuições a dois campos extremamente atuais: a nanotecnologia e a computação quântica. Curioso que essas ideias foram inicialmente apresentadas em conferências. A primeira, "There is plenty of room at the bottom - an invitation for a new field of physics", foi apresentada numa sessão plenária da Sociedade Americana de Física, em Pasadena, ano de 1959. Segundo Schulz, ela é "considerada como a primeira palestra sobre a tecnologia e a engenharia na escala atômica" por um site importante da área e a publicação se tornou uma das mais citadas da obra de Feynman.

Em um keynote address de uma conferência no MIT, sobre "Física da Computação", Feynman sugere a possibilidade de construir um "computador quântico". De início, teoriza sobre o uso de computadores para simular sistemas quânticos, discutindo inicialmente as possibilidades do ponto de vista da computação e da física. Que tipo de computador precisamos? Ele opta pelos autômatos celulares, mas sugere sistemas computacionais que mimetizem sistemas da natureza. E passa a discutir como simular o tempo e as probabilidades quânticas, fornecendo um exemplo de que é impossível obter os resultados da mecânica quântica usando um dispositivo clássico universal. Nessa conferência, Feynman aponta as bases da computação quântica. Em seguida, se interessa em lecionar sobre esse tema durante vários anos no Caltech e suas palestras resultaram no Feynman Lectures on Computation. [22] Mas para fins de tecnologia quântica, para a fabricação de um processador quântico, o problema da dissipação quântica não pode ser ignorado. Caldeira mostra com profundidade as contribuições de Feynman para cada uma das áreas de dissipação e computação quântica.

\section{Feynman e a matéria condensada}

Feynman se interessou pela teoria do hélio líquido porque acreditava que o problema deveria ser tratado por uma teoria microscópica obtida a partir de primeiros princípios. Mesmo reconhecendo as contribuições relevantes e o sucesso da teoria fenomenológica de Landau, ele apontou algumas de suas fraquezas. Ele acreditava que uma teoria do hélio líquido II deveria ser formulada com base nos princípios da mecânica quântica e da equação de Schrödinger do sistema. Depois de curta visita ao Bra- 
si ${ }^{6}$ Feynman participou da Conference on Theoretical Physics, em Tóquio, onde enfrentou, pela primeira vez, a comunidade internacional no campo do hélio líquido e da mecânica estatística, sendo sua apresentação elogiada por Lars Onsager. Sua mais notável contribuição apareceu no começo de 1954, consistindo na determinação do espectro de energia das excitações elementares do hélio líquido. Mais tarde, Feynman apresentou sua teoria da vorticidade quantizada. As importantes contribuições de Feynman são analisadas por Paulo Farinas dentro do contexto do que se conhecia na época, isto é, em especial as contribuições de Landau.

Feynman investiu seu talento criativo na solução do problema dos pólarons, as excitações elétron-fônon em cristais iônicos, motivado para encontrar alguma luz no problema mais desafiador e atual, a supercondutividade. Além disso, o problema poderia ser facilmente resolvido (por ele!) usando seu método de cálculo desenvolvido na QED. Fracassou na tentativa, mas seu cálculo variacional da energia e da massa efetiva do pólaron forneceu os melhores resultados comparados com todas as demais aproximações até hoje.

\section{Feynman e as partículas elementares}

Os resultados sobre a estrutura dos núcleons obtidos no Stanford Linear Accelerator Center (SLAC), ao final da década de 60, forneciam evidências de estruturas carregadas pontuais dentro do núcleon. Feynman fez uma visita ao SLAC, interpretou os dados da seção de choque de espalhamento lépton-núcleon e concluiu que os resultados indicavam a presença de constituintes elementares dotados de carga elétrica dentro dos núcleons, os pártons de Feynman, assim contado pelo Carlos Escobar, físico brasileiro atualmente no Fermilab. 7 em seu artigo sobre o modelo de pártons. A ideia básica de Feynman é representar o espalhamento inelástico elétron-núcleon como o espalhamento quase-livre de partículas pontuais dentro do próton visto por um referencial em que o próton tem grande momento e o espalhamento é profundamente inelástico. Poucos anos antes, Gell-Mann e Zweig haviam proposto independentemente o modelo de quarks que explicava bem propriedades estáticas dos hádrons, mas que não se imaginava que pudesse ser usado para explicar processos dinâmicos. Embora haja, de fato, uma estreita relação entre eles, esses dois conceitos não são, no entanto, idênticos.

Quarks x pártons foi um ingrediente a mais na disputa acadêmica de Gell-Mann e Feynman como em episódios narrados por Tony Hey. [10]. Aos interessados em aprender mais, sugiro ler as notas de aula do curso de Jiri Chyla [23].

\footnotetext{
6 Feynman visitou o Brasil em julho-agosto de 1953, logo após submeter os dois primeiros artigos sobre a transição lambda do hélio líquido (abril e junho, respectivamente).

${ }^{7}$ A tese de doutorado de Escobar, obtido em Cambridge em 1978, é intitulada Application of the Parton Model to Large Transverse Momentum Processes.
}

\section{Considerações finais}

Há inúmeros artigos, vídeos, postagens em redes sociais, celebrando Feynman no dia 11 de maio de 2018, data em que faria 100 anos. No entanto, uma leitura do número especial da Physics Today, de fevereiro de 1989, é recomendada especialmente por conter depoimentos pessoais sobre a obra de Feynman de grandes físicos que conviveram com ele. Existe uma enorme literatura disponível que o leitor mais interessado pode consultar. Na literatura em português, recomendo a biografia de Feynman por Bassalo e Caruso [24], e o livrinho de Rogério Rosenfeld em que é descrito o difícil relacionamento de Feynman e Gell-Mann [25].

Com essa edição especial, pesquisadores brasileiros, por meio da RBEF, prestam homenagem ao grande e cultuado físico que amava e tantas vezes visitou o Brasil, tanto para fazer física como se divertir no Carnaval carioca.

Agradecimentos calorosos a todos os colaboradores convidados e, em especial, ao Silvio Salinas, Editor Chefe da RBEF, que tornaram possível essa edição especial.

\section{Referências}

[1] D. Kaiser, Drawing Theories Apart: The Dispersion of Feynman Diagrams in Postwar Physics (University of Chicago Press, Chicago, 2005).

[2] R.P. Feynman e A. Hibbs, Quantum Mechanics and Path Integrals (McGraw Hill, New York, 1965).

[3] D. Styer, Resonance 16, 849 (2011).

[4] R.P. Feynman, R.B. Leighton e M. Sands, The Feynman Lectures on Physics (Addison-Wesley, Boston, 1965).

[5] R.P. Feynman, in: Quantum Implications: Essays in Honour of David Bohm, editado por F.D. Peat e B. Hiley (Routledge, Londres, 1991), p. 235.

[6] R.P. Feynman, Sobre as leis da física (Contraponto, Rio de Janeiro, 2012).

[7] R.P. Feynman, O senhor está brincando, Sr. Feynman!: as estranhas aventuras de um físico excêntrico (Elsevier, São Paulo, 2006).

[8] L. Mlodinow, O arco íris de Feynman (Sextante, Rio de Janeiro, 2005).

[9] A.J.G. Hey, Physics Today 49, 44 (1996).

[10] L. Brown. Resonance 19, 874 (2011).

[11] R.P. Feynman, in: The pleasure of findings things out, editado por J. Robbins (Penguin Books, Londres, 2001).

[12] J. Gleick, Genius: The Life and Science of Richard Feynman (Open Road Media, New York, 2011).

[13] M.A. Moreira, Aprendizagem significativa: a teoria e textos complementares (Editora Livraria da Física, São Paulo, 2011).

[14] T. Pipes, Learning From the Feynman Technique, disponível em https://bit.ly/2s9Yr6F

[15] T. Frank, How to Learn Faster with the Feynman Technique (Example Included), disponível em https: //bit.1y/2J0Rz66.

[16] R.P. Feynman, R.B. Leighton e M. Sands, Feynman - Lições de Física: edição definitiva (Bookman, Porto Alegre, 2008). 
[17] R.P. Feynman, Rev. Mod. Phys. 20, 367 (1948).

18] J. Mehra, The Beat of a Different Drum: The Life and Science of Richard Feynman (Oxford University Press, New York, 1994).

[19] R.P. Feynman, Feynman thesis, a new approach to quantum theory, editado por L. Brown (World Scientific Publishing Company, New Jersey, 2005).

[20] S. Tuleja, B. Gazovic e A. Tomori, Am. J. Phys. 75, 240 (2007).

[21] A. Pais, Inward Bound: Of Matter and Forces in the Physical World (Clarendon Press, Wotton-under-Edge, 1988)

[22] T. Hey, Contemp. Phys. 40, 257 (1999)

[23] J. Chyla, Quarks, partons and quantum chromodynamics, disponível em www-hep2.fzu.cz/ chyla/ lectures/text.pdf

[24] J.M.F. Bassalo e F. Caruso, Feynman (Editora Livraria da Física, São Paulo, 2013).

[25] R. Rosenfeld, Feynman e Gell-Mann (Editora Odysseus, São Paulo,2003). 